\title{
Associations of Actigraphy-derived Rest-activity Patterns and Circadian Phase Parameters With Clinical Symptoms and Polysomnography Results in Patients With Chronic Insomnia
}

\section{Hyun Woong Roh}

Ajou University School of Medicine

Su Jung Choi

Sungkyunkwan University

Hyunjin Jo

Samsung Biomedical Research Institute, Samsung Medical Center, Sungkyunkwan University School of Medicine

\section{Dongyeop Kim}

Samsung Biomedical Research Institute, Samsung Medical Center, Sungkyunkwan University School of Medicine

Jung-gu Choi

Yonsei University

\section{Sang Joon Son}

Ajou University School of Medicine

\section{Eun Yeon Joo ( $\nabla$ eunyeon.joo@gmail.com )}

Samsung Biomedical Research Institute, Samsung Medical Center, Sungkyunkwan University School of Medicine

\section{Research Article}

Keywords: actigraphy-derived rest-activity patterns, circadian phase, clinical symptoms, polysomnography, chronic insomnia, patients, results

Posted Date: October 29th, 2021

DOI: https://doi.org/10.21203/rs.3.rs-1011658/v1

License: (c) (1) This work is licensed under a Creative Commons Attribution 4.0 International License. Read Full License 


\section{Abstract}

We explored the associations of actigraphy-derived rest-activity patterns and circadian phase parameters with clinical symptoms and level 1 polysomnography (PSG) results in patients with chronic insomnia to evaluate the clinical implications of actigraphy-derived parameters for PSG performance. Seventy-five participants underwent actigraphy assessments and level 1 PSG. Exploratory correlation analyses between parameters derived from actigraphy, PSG, and clinical assessments were performed. Study participants were classified into two groups, and group differences were investigated after adjusting for potential covariates. Participants with poorer rest-activity patterns on actigraphy (low inter-day stability and high intra-daily variability) exhibited higher insomnia severity index scores compared to participants with better rest-activity patterns. No between-group differences in PSG parameters were observed. Latephase participants (late least active 5-h and most active 10-h onset times) exhibited higher insomnia severity scores, longer sleep latency, longer rapid eye movement latency, and lower apnea-hypopnea index compared to early-phase participants. These associations remained significant even after adjusting for potential covariates. Some actigraphy-derived rest-activity patterns and circadian phase parameters were significantly associated with clinical symptoms and PSG results, suggesting a possible adjunctive role of actigraphy-derived rest-activity patterns and circadian phase parameters in deriving plans for PSG lightsoff time and assessing the possible insomnia pathophysiology.

\section{Introduction}

The circadian clock is a system that enables organisms to adapt to external changes in the environment over a 24-hour period and is a major determinant of rest-activity patterns, sleepiness, and alertness in humans. ${ }^{1}$ Growing evidence suggests that homeostatic sleep drive (Process $\mathrm{S}$ ) and the circadian pacemaker (Process $\mathrm{C}$ ) reciprocally interact to regulate the sleep-wake cycle. ${ }^{2}$ For example, animals lacking core clock machinery, such as Bmal1 or Clock genes, exhibit impairments in the circadian pacemaker and changes in sleep and quality, such as altered EEG delta power. ${ }^{3-5}$ Alterations in restactivity patterns and circadian phase have also been reported in patients with bipolar disorder, Parkinson's disease, and Alzheimer's disease. ${ }^{6-10}$ Indeed, circadian rhythm disruptions and sleep difficulties are frequently observed in patients with these conditions, suggesting pathophysiological roles for sleep and circadian alterations in disease initiation and progression. ${ }^{11}$ Nevertheless, rest-activity patterns, circadian phase alterations, and their clinical implications in patients with insomnia remain poorly characterized.

Polysomnography (PSG) is considered the gold standard for objectively evaluating sleep quality and quantity. ${ }^{12}$ PSG provides an accurate measure of wake and sleep times, as well as respiratory function, muscle activity, heart physiology, and sleep stages. However, level 1 PSG is typically performed for one night in a specialized setting, regardless of patients' lifestyle, such as sleep environment, which may contribute to poor ecological validity. ${ }^{13}$ Sleep diaries and/or questionnaires are commonly used to identify patients' lifestyle and circadian phase. ${ }^{14,15}$ Actigraphy has emerged as a major assessment tool 
in sleep research and sleep medicine over the last two decades. ${ }^{16,17}$ In this context, patients' rest-activity patterns and circadian phase derived from several days of actigraphy assessment may be helpful for performing and/or interpreting level 1 PSG in clinical practice. Several studies have compared PSGderived sleep parameters with actigraphy-derived parameters such as total sleep time and wakefulness

after sleep onset. ${ }^{18-20}$ However, to the best of our knowledge, no study to date has evaluated the clinical significance of actigraphy-derived rest-activity patterns and circadian phase parameters for the interpretation of level 1 PSG results.

In this study, we performed clinical evaluation, actigraphy assessments, and level 1 PSG in 75 participants with chronic insomnia. We investigated the relationships of actigraphy-derived rest-activity patterns and circadian phase with clinical symptoms and PSG parameters of participants with chronic insomnia in our sleep clinic in order to evaluate the possible associations and clinical implications of actigraphy-derived parameters for the performance and interpretation of PSG.

\section{Results}

\section{Demographic characteristics, rest-activity patterns, circadian phase, and PSG results}

Demographic characteristics, rest-activity patterns, and circadian phase according to actigraphy, PSG results, and clinical symptom scores of the study participants are listed in Table 1. In brief, the median (IQR) age was 58 (51-65) years and BMI was 22.2 (20.4-24.2). Of the 75 participants, 59 (78.7\%) were female. Of the participants, 66 (88.0\%) wore the actigraphy device over the entire 7 days, and nine (12.0\%) reported 1-2 missing days during the recording period. Rest-activity pattern variables (IS and IV) and circadian phase variables (L5 and M10 onset times) were extracted from the actigraphy measurements. The mean and median values of these parameters did not deviate substantially from our previous findings in elderly individuals with cognitive impairments and other studies assessing similar parameters. ${ }^{7,8,21}$ PSG results indicated that total sleep time and wake after sleep onset were approximately $361.9 \mathrm{~min}$ and $14.4 \%$, respectively.

Table 1. Demographic characteristics, rest-activity patterns, circadian phase, and level 1 PSG parameters of study participants. 
Variables ${ }^{a}$

Age, year

Female, No. (\%)

Height, cm

Weight, kg

Body mass index

Actigraphy recording days, No. (\%)

- 5 days

-6 days

-7 days

Rest-activity patterns and circadian phase on actigraphy

- IS, day to day consistency

$0.56(0.46-0.64)$

- IV, fragmentation of activity

- L5 onset time, rest phase, time

- M10 onset time, active phase, time

\section{Polysomnography (level 1) results}

- Total sleep time, min $361.9(61.6)$

- Sleep latency, min

$10.5(6.5-28.5)$

- REM latency, min

97.5 (74.0-154.0)

- Sleep efficiency, \%

- Wakefulness after sleep onset, \%

- Sleep stage N1, \%

- Sleep stage N2, \%

- Sleep stage N3, \%
$0.81(0.20)$

$24.5(1.0)$

$9.5(2.1)$
82.0 (74.1-88.2)

$14.4(8.0-23.2)$

14.4 (9.7-18.9)

$61.4(10.0)$

$0.8(0.0-3.6)$
All participants $(n=75)$

$58(51-65)$

59 (78.7)

158.0 (154.0-163.0)

$56.0(51.0-62.0)$

$22.2(20.4-24.2)$

$6(8.0)$

$66(88.0)$

a Values are presented as mean (SD) for normally distributed continuous variables, median (IQR) for non-normally distributed continuous variables, and number (\%) for categorical variables.

${ }^{b}$ Five participants did not complete the insomnia severity index $(n=70)$, and one participant did not complete the Epworth sleepiness scale $(n=74)$.

IQR, interquartile range; IS, interday stability; IV, intradaily stability; L5 onset time, least active 5-h onset time; M10 onset time, most active 10-h onset time; SD, standard deviation. 
- Sleep stage REM, \%

- Apnea Hypopnea Index

\section{Clinical symptom score ${ }^{b}$}

- Insomnia severity index

- Epworth sleepiness scale

a Values are presented as mean (SD) for normally distributed continuous variables, median (IQR) for non-normally distributed continuous variables, and number (\%) for categorical variables.

${ }^{b}$ Five participants did not complete the insomnia severity index $(n=70)$, and one participant did not complete the Epworth sleepiness scale $(n=74)$.

IQR, interquartile range; IS, interday stability; IV, intradaily stability; L5 onset time, least active 5-h onset time; M10 onset time, most active 10-h onset time; SD, standard deviation.

\section{Exploratory correlation analyses of rest-activity patterns, circadian phase, PSG parameters, and clinical symptom scores}

To explore the associations among rest-activity patterns, circadian phase, PSG parameters, and clinical symptom scores, Pearson or Spearman correlation analyses were performed based on the distribution of variables (Table 2). As expected, a negative association was noted between actigraphy-derived IS and IV $(P<0.001)$, and a positive association was observed between L5 and M10 onset times $(P<0.001)$. In addition, actigraphy-derived $L 5$ onset time, indicative of the rest phase, was negatively associated with AHI $(P=0.05)$ and positively associated with REM latency $(P=0.006)$ and ISI $(P=0.03)$. M10 onset time, indicative of the active phase, was positively associated with REM latency $(P=0.04)$. These results suggest that the actigraphy-derived circadian phase might be associated with insomnia severity and some PSG parameters such as AHI and REM latency in patients with insomnia disorder.

Table 2. Exploratory correlation analyses among rest-activity patterns, circadian phase, level 1 PSG parameters, and clinical symptom scores ${ }^{a}$. 


\begin{tabular}{|c|c|c|c|c|c|c|c|c|c|c|c|c|c|c|c|c|}
\hline & \multicolumn{2}{|c|}{\begin{tabular}{|c|}
$\begin{array}{c}\text { Actigraphy } \\
\text { (Rest-activity patterns) }\end{array}$ \\
\end{tabular}} & \multicolumn{2}{|c|}{$\begin{array}{c}\text { Actigraphy } \\
\text { (Circadian phase) }\end{array}$} & \multicolumn{10}{|c|}{ Polysomnography (level 1) parameters } & \multicolumn{2}{|c|}{$\begin{array}{l}\text { Clinical symptom } \\
\text { scores }\end{array}$} \\
\hline & IS & IV & \begin{tabular}{|c|} 
L5 \\
onset time
\end{tabular} & \begin{tabular}{|c|} 
M10 \\
onset time
\end{tabular} & $\begin{array}{c}\text { Total } \\
\text { sleep time }\end{array}$ & $\begin{array}{c}\text { Sleep } \\
\text { latency }\end{array}$ & $\begin{array}{c}\text { REM } \\
\text { latency }\end{array}$ & $\mathrm{SE}, \%$ & WASO $\%$ & N1 \% & $\mathrm{N} 2 \%$ & N3 $\%$ & REM \% & AHI & ISI & ESS \\
\hline IV & $-0.436^{* * *}$ & 1 & & & & & & & & & & & & & & \\
\hline L5 onset time & $-0.285^{*}$ & 0.178 & 1 & & & & & & & & & & & & & \\
\hline M10 onset time & -0.216 & 0.101 & $0.379 * \cdots$ & 1 & & & & & & & & & & & & \\
\hline Total sleep time, min & -0.045 & -0.096 & 0.042 & 0.097 & 1 & & & & & & & & & & & \\
\hline REM latency, min & -0.055 & $0.279^{*}$ & $0.315^{* *}$ & $0.242 *$ & $-0.233^{*}$ & $0.256^{*}$ & 1 & & & & & & & & & \\
\hline SE, \% & -0.017 & -0.086 & -0.053 & 0.101 & $0.802 \cdots \cdots$ & $-0.464^{* \cdots \cdots}$ & $-0.302 * *$ & 1 & & & & & & & & \\
\hline WASO, $\%$ & 0.072 & 0.116 & 0.050 & -0.124 & $-0.790^{* * *}$ & 0.181 & $0.267^{*}$ & $-0.906 * * *$ & 1 & & & & & & & \\
\hline N1 sleep, \% & -0.022 & 0.162 & 0.094 & -0.058 & -0.163 & -0.124 & 0.224 & -0.158 & $0.317 * *$ & 1 & & & & & & \\
\hline $\mathrm{N} 2$ sleep, $\%$ & 0.197 & -0.125 & -0.058 & 0.085 & $=0.102$ & 0.159 & 0.224 & -0.031 & -0.109 & $-0.585 * * *$ & 1 & & & & & \\
\hline N3 sleep, \% & 0.011 & -0.026 & 0.139 & 0.096 & 0.054 & 0.005 & -0.176 & 0.090 & -0.117 & $-0.265^{\circ}$ & -0.144 & 1 & & & & \\
\hline REM sleep, $\%$ & -0.062 & -0.007 & -0.166 & -0.172 & $0.250^{*}$ & -0.086 & $-0.579 * * *$ & $0.248^{*}$ & -0.177 & -0.125 & $-0.475^{* * *}$ & -0.004 & 1 & & & \\
\hline $\mathrm{AHI}$ & 0.005 & -0.017 & $-0.226^{*}$ & -0.189 & 0.004 & $-0.281^{*}$ & -0.060 & 0.026 & 0.097 & $0.237^{*}$ & -0.118 & $-0.258^{*}$ & -0.053 & 1 & & \\
\hline
\end{tabular}

a Pearson correlation test was performed between two normally distributed continuous variables. Otherwise, Spearman rank correlation test was performed. Red and blue boxes indicate significant positive and negative associations, respectively.

${ }^{b}$ Five participants did not complete the insomnia severity index $(n=70)$, and one participant did not complete the Epworth sleepiness scale $(n=74)$.

AHI, apnea-hypopnea index; ESS, Epworth sleepiness scale; IS, interday stability; ISI, insomnia severity index; IV, intradaily stability; L5 onset time, least active 5-h onset time; M10 onset time, most active 10-h onset time; REM, rapid eye movement; WASO, wakefulness after sleep onset.

\section{Data-driven group classification based on actigraphy- derived rest-activity patterns and circadian phase}

Hierarchical clustering analyses were performed for the actigraphy data-driven group classification of participants for the following reasons. First, rest-activity patterns and circadian phase alterations may only occur in a subset of patients with chronic insomnia in sleep clinics. Second, critical values for actigraphy-derived rest-activity patterns or circadian phase parameters have not been suggested to date. Third, using a data-driven clustering approach, two rest-activity pattern variables, IS and IV, or two circadian phase variables, L5 and M10, can be simultaneously considered. The 75 participants were classified into two groups based on their rest-activity patterns and circadian phase variables (Figure 2). Group classification according to rest-activity patterns (IS and IV) clustered 17 participants with low IS and high IV (Group 1), and 58 participants with high IS and low IV (Group 2). Group classification according to circadian phase ( $\mathrm{L} 5$ and M10 onset times) clustered 37 late-phase participants (group L) and 38 early-phase participants (group E).

\section{Differences in PSG parameters and clinical symptom scores according to rest-activity pattern variables}

To investigate between-group differences and their clinical implications according to rest-activity pattern variables (IS and IV), PSG parameters and clinical symptom scores were compared between groups. Participants with poorer rest-activity patterns (Group 1, low IS, and high IV) had significantly higher ISI 
scores in the clinical assessment $(P=0.04$; Figure $3 a)$. This difference remained significant after adjusting for age, sex, BMI, and lights-off time by applying ANCOVA (estimated marginal means [standard error], Group 1: 20.8 [1.3] vs Group 2: 17.7 [0.6]; P = 0.04). No significant between-group differences were noted in other PSG parameters or ESS scores.

\section{Differences in PSG parameters and clinical symptom scores according to circadian phase variables}

To investigate between-group differences and their clinical implications according to circadian phase variables (L5 and M10 onset times), PSG parameters and clinical symptom scores were compared between groups. Late-phase participants (Group L) had significantly higher ISI scores in the clinical assessment $(P=0.03$; Figure $3 b)$. This difference remained significant after adjusting for age, sex, $B M I$, and lights-off time by applying ANCOVA (estimated marginal means [standard error], Group L: 19.6 [0.8] vs Group E: 17.0 [0.8]; $P=0.03$ ). In addition, late-phase participants (Group L) exhibited significantly longer sleep latency $(P=0.04$; Figure $3 c)$, REM latency $(P=0.04$; Figure $3 d)$, and lower $A H I(P=0.02$; Figure $3 \mathrm{e}$ ) on PSG. Considering the skewed distribution of parameters and clinical implications of sleep latency, REM latency, and $\mathrm{AHI}$, logistic regression analysis was performed using conventional cut-off scores after adjusting for age, sex, BMI, and lights-off time. Conventional cut-off scores were defined as 10 min for sleep latency, 150 min for REM latency, and 5 min for AHI. Compared to early-phase participants (Group E), late-phase participants (Group L) had adjusted odds ratios (ORs) of 5.8 (95\% confidence interval $[\mathrm{Cl}], 1.8-18.2, \mathrm{P}=0.003)$ for long sleep latency, $4.0(95 \% \mathrm{Cl}, 1.2-13.6, \mathrm{P}=0.04)$ for long REM latency, and $0.26(95 \% \mathrm{Cl}, 0.07-0.94, \mathrm{P}=0.04)$ for abnormal $\mathrm{AHI}$.

\section{Discussion}

The present study explored the associations of actigraphy-derived rest-activity patterns and circadian phase parameters with clinical symptoms and level 1 PSG results in 75 patients with chronic insomnia at a sleep clinic. Exploratory correlation analyses revealed significant associations of actigraphy-derived circadian phase parameters (L5 and M10 onset times) with insomnia severity and level 1 PSG parameters such as AHI or REM latency. The 75 subjects were further categorized into two groups based on rest-activity patterns or circadian phase using data-driven hierarchical clustering analysis. The analysis revealed that participants with poorer rest-activity patterns (low IS and high IV) exhibited higher insomnia severity scores compared to participants with better rest-activity patterns (high IS and low IV). In addition, late-phase participants (late L5 and M10 onset times) exhibited higher insomnia severity scores, longer sleep latency, longer REM latency, and lower AHI compared to early-phase participants (early L5 and M10 onset times). These associations remained significant even after adjusting for potential covariates including age, sex, BMI, and lights-off time.

There are several implications that arise from the present study. We did not identify strong relationships between actigraphy-derived rest-activity pattern parameters (IS and IV) and level 1 PSG results in patients with chronic insomnia. IS and IV are the most well-described rest-activity pattern parameters that 
measure the similarity of one 24-hour period to the next and the strength of consolidation of the restactivity rhythm within a 24-hour period, respectively. Several studies have reported possible associations of IS and IV with the differential diagnosis, severity, and pathophysiology of diseases such as Alzheimer's disease, Parkinson's disease, bipolar disorder, and posttraumatic stress disorder. ${ }^{8,9}$ Changes in restactivity patterns and circadian rhythm disruptions have been suggested to play pathophysiological roles in these conditions ${ }^{11,22}$ as well as in the initiation and progression of insomnia disorder. ${ }^{12,22-24}$ In the present study, we only identified a weak nonparametric positive association between IV and REM latency. However, following categorization of participants into two groups based on IS and IV (Group 1 and Group 2), no significant difference in REM latency was observed between the two groups (data not shown, $P=$ 0.07, Mann-Whitney U test). In addition, other major level 1 PSG parameters such as TST, SL, WASO, N1/N2/N3/REM proportion, and AHI were not significantly associated with IS or IV. These findings are not entirely unexpected given the multifactorial pathophysiology of insomnia disorder. ${ }^{25,26}$ Although there is currently no consensus on the proportion of insomnia patients with circadian rhythm disruptions, the normal distribution of IS and IV parameters in our study suggests that only a subset of patients with chronic insomnia have abnormal rest-activity patterns and circadian rhythms. Another possibility is that actigraphy-derived rest-activity pattern parameters (IS and IV) are factors independent of insomnia severity, regardless of PSG parameters. Indeed, there is a discrepancy between objective measurements of sleep and subjective insomnia. ${ }^{27}$ This common misperception of sleep in patients with insomnia is problematic because patients perceive that they are getting insufficient sleep. Therefore, patients with insomnia that have sleep misperception might tend to spend more time on sleep than necessary, even during the daytime, which could result in inappropriate and irregular rest-activity patterns.

Participants with late circadian phase on actigraphy (Group L) presented with more complaints regarding insomnia symptoms and longer sleep and REM latency in PSG. Although this association may seem obvious, our findings have important clinical implications for the performance and interpretation of PSG in sleep clinics. The ecological validity of PSG has been questioned due to the disparity between the specialized environment required for level 1 PSG and patients' home setting. For example, longer sleep or REM latency on PSG is typically considered to indicate difficulties with sleep initiation in the daily lives of patients. However, it could also be due to delayed circadian phase or discomfort induced by the numerous devices required for level 1 PSG on the day of assessment. Differentiating these factors in clinical practice is critical, but may be challenging due to limited information about the patients' circadian phase or degree of discomfort in PSG. In the present study, actigraphy-derived circadian phase was calculated, revealing that patients with delayed circadian phase had more abnormal sleep and REM latency based on conventional cut-off scores. Thus, our findings imply a possible adjunctive role of actigraphy-derived circadian phase information for the performance and interpretation of PSG. For instance, patients with early circadian phase on actigraphy but abnormal sleep and REM latency on PSG may have more severe sleep initiation difficulties in daily life or feel more uncomfortable during PSG. In contrast, for patients with delayed circadian phase on actigraphy, our findings suggest that sleep physicians should assess patients' PSG lights-off time based on patients' routine sleep schedule reports and actigraphy-measured circadian phase before PSG. Theoretically, PSG lights-off time should be 
consistent with patients' routine sleep schedule. However, in clinical practice, there may be discrepancies between PSG lights-off time and patients' routine sleep schedule or circadian phase due to limited and imprecise information reported by patients. We conjecture that actigraphy-measured circadian phase may provide additional adjunctive information to sleep physicians for deriving plans for PSG lights-off time. In addition, sleep difficulties of patients with delayed circadian phase on actigraphy and abnormal sleep and REM latency on PSG may be due to initiation of sleep that is too early for patients' circadian phase. Indeed, Flynn-Evans et al. analyzed dim light melatonin onset (DLMO) with sleep logs in 79 patients with insomnia and reported that a substantial proportion (10-22\%) of patients with insomnia initiated sleep too early for their circadian phase. ${ }^{28}$ For these cases, reassessing circadian rest-activity phase in daily life and applying chronotherapy, melatonin, and/or timed light exposure are potential options for addressing sleep difficulties. ${ }^{29-31}$

Finally, participants with early circadian phase on actigraphy (Group E) exhibited a slightly higher AHI on PSG. This group difference could be caused by confounding factors. Indeed, participants in Group E were older than subjects in Group L (mean age of 60.6 and 53.7 years in Groups E and L, respectively), but no differences in sex or BMI were noted. However, after adjusting for possible covariates including age, subjects in Group E were still significantly more prone to have abnormal AHI on PSG. One large epidemiologic sleep study reported increased AHI values in morning- and evening-type individuals after stratifying the sample by $\mathrm{BMI}$ and age. ${ }^{32}$ The association between higher $\mathrm{AHI}$ values and early circadian phase observed in our study may reflect the protective role of a normal circadian phase in obstructive sleep apnea severity.

This study has several limitations. This study was conducted using a relatively small sample size. In addition, it should be noted that participants in our study were predominantly middle-aged to older adults. These points may limit the generalizability of our results at a population level. Furthermore, our study was cross-sectional; hence, causal relationships could not be conclusively demonstrated.

In conclusion, this study investigated the associations of actigraphy-derived rest-activity patterns and circadian phase with level 1 PSG results in patients with chronic insomnia. Our analysis revealed that participants with poorer rest-activity patterns (low IS and high IV) on actigraphy assessment exhibited higher insomnia severity scores compared to subjects with better rest-activity patterns (high IS and low IV). In addition, late-phase participants (late L5 and M10 onset times) exhibited higher insomnia severity scores, longer sleep latency, longer REM latency, and lower AHI compared to early-phase participants. Notably, these associations remained significant even after adjusting for covariates. We highlight the clinical implications and the possible adjunctive role of actigraphy-derived rest-activity patterns and circadian phase parameters for the performance and interpretation of PSG in patients with insomnia. Future studies using a larger sample size and repeated assessments of actigraphy and PSG are warranted.

\section{Methods}




\section{Participants}

This retrospective cross-sectional study used data from the database of a single university-affiliated hospital located in Seoul, South Korea, from May 2016 to January 2020. All patients in the database underwent overnight PSG and monitoring of actigraphy for at least 7 days from the night of PSG. In total, 124 participants diagnosed with chronic insomnia $(\mathrm{Cl})$ based on clinical symptoms and criteria of the Diagnostic and Statistical Manual of Mental Disorders (DSM-IV-TR) and International Classification of Sleep Disorders, Third Edition (ICSD-2) were consecutively selected. Inclusion criteria were individuals aged over 20 years with subjective sleep complaints. Exclusion criteria were as follows: non-Koreanspeaking foreigners; shift workers; abnormal sleep-wake disorders; other sleep disorders, such as restless legs syndrome, narcolepsy, rapid eye movement (REM) sleep behavior disorder, or periodic limb movement disorder, severe medical, neurological (neurodegenerative diseases, epilepsy, head injury), or psychiatric diseases (psychosis, current intake of antidepressants for diagnosed depression), and alcohol or illicit drug abuse or current intake of psychoactive medications. A total of 49 participants were excluded due to insufficient actigraphy data (less than 4 days, $n=32$ ), shift worker or irregular sleep-wake rhythm $(n=13)$, and insomnia due to restless leg syndrome with or without definite periodic limb movement disorder $(n=4)$. A final total of 75 participants were enrolled in this study.

\section{Standard protocol approval, registration, and patient consent}

This study was approved by the Institutional Review Board of Samsung Medical Center (SMC IRB No. 2021-04-021). The need for patient informed consent was waived due to the retrospective nature of the study. This study was performed in accordance with relevant guidelines and regulations of the IRB.

\section{Measurement of rest-activity patterns and circadian phase using actigraphy}

Participants were instructed to wear a research-grade triaxial accelerometer Actiwatch 2 (Phillips Respironics, Murrysville, PA, USA) on their non-dominant wrist for at least 7 days prior to the night of PSG while performing their usual daily activities in a home setting. Activity counts in 1-min epochs from the first 7 consecutive days of data, commencing at midnight, were processed to calculate rest-activity patterns and circadian phase variables.

Rest-activity patterns and circadian phase parameters were extracted using nonparametric analysis of actigraphy data. Nonparametric analysis does not adopt a priori assumptions about the waveform of daily activity; rather, it calculates variables based on raw activity counts. ${ }^{33}$ The following parameters were calculated from the non-parametric analysis: (1) inter-daily stability (IS), which generally represents the strength of coupling of a rhythm to environmental zeitgebers; (2) intra-daily variability (IV), which generally represents activity fragmentation in a day; (3) least active 5-h (L5) onset time; and (4) most active 10-h (M10) onset time. Actigraphy data was handled using Actiware version 5.7 software and 
'nparACT' package for R Statistical Software. ${ }^{34,35}$ The distributions and correlations of four rest-activity patterns and circadian phase variables are presented in Figure 1.

\section{Polysomnography}

PSG measurements were recorded during one night of observation with standard electrodes and sensors using Embla N7000 (Medcare Flaga, Iceland). The following PSG parameters were measured and collected: total sleep time (TST), sleep latency, REM latency, wakefulness after sleep onset (WASO), percent time in each sleep stage (N1, N2, N3, and REM stages), and apnea-hypopnea index (AHI). Participants completed the Insomnia Severity Index (ISI) and Epworth Sleepiness Scale (ESS) questionnaires to measure the severity of subjectively reported insomnia symptoms and daytime sleepiness, respectively. 36,37

\section{Statistical analysis}

Continuous variable data were reported as mean and standard deviation (SD) or median and interquartile range (IQR) after verifying the normality of the data distribution using the Shapiro-Wilk test. To explore possible associations between rest-activity patterns, circadian phase, PSG parameters, and clinical symptom scores, Pearson correlation analysis was performed for two normally distributed continuous variables. If one or both variables did not have a normal distribution, nonparametric Spearman correlation analysis was performed. Hierarchical clustering analyses were performed using Ward's linkage algorithm with Euclidean distances for the actigraphy data-driven group classification of participants. For group comparisons, the Student's t-test or Mann-Whitney $U$ test was used for variables exhibiting a normal or non-normal distribution, respectively. Analysis of covariance (ANCOVA) was performed to examine the significance of observed associations after adjusting for potential covariates including age, sex, body mass index (BMI), and lights-off time. Considering the skewed distribution and clinical implications of sleep latency, REM latency, and AHI on PSG, logistic regression analysis was performed using conventional cut-off scores after adjusting for age, sex, BMI, and lights-off time.

\section{Declarations}

\section{Acknowledgements}

The authors thank the study participants, staff, and investigators for their contributions to this study.

\section{Author Contributions}

HWR, SJC, and EYJ formulated the conception and design of this work. HWR, SJC, HJ, DK, and JC contributed to data acquisition. All authors contributed to the analysis and interpretation of the data. HWR, SJC, SJS, and EYJ drafted the manuscript and figures. All authors made critical revisions and approved the final version of the manuscript.

\section{Competing interests}


The authors declare no competing interests.

\section{Funding sources}

This research was supported by Samsung Medical Center Grant (OTC1190671) and by a grant from the Korea Health Technology R\&D Project via the Korea Health Industry Development Institute (KHIDI), funded by the Ministry of Health \& Welfare, Republic of Korea (grant number: HR21C0885).

\section{References}

1. Reppert, S. M. \& Weaver, D. R. Coordination of circadian timing in mammals. Nature 418, 935-941, doi:10.1038/nature00965 (2002).

2. Borbely, A. A., Daan, S., Wirz-Justice, A. \& Deboer, T. The two-process model of sleep regulation: a reappraisal. J Sleep Res 25, 131-143, doi:10.1111/jsr.12371 (2016).

3. Franken, P. A role for clock genes in sleep homeostasis. Curr Opin Neurobio/ 23, 864-872, doi:10.1016/j.conb.2013.05.002 (2013).

4. Haque, S. N., Booreddy, S. R. \& Welsh, D. K. Effects of BMAL1 Manipulation on the Brain's Master Circadian Clock and Behavior. Yale J Biol Med 92, 251-258 (2019).

5. Charrier, A., Olliac, B., Roubertoux, P. \& Tordjman, S. Clock Genes and Altered Sleep-Wake Rhythms: Their Role in the Development of Psychiatric Disorders. Int J Mol Sci 18, doi:10.3390/ijms18050938 (2017).

6. Smagula, S. F., Gujral, S., Capps, C. S. \& Krafty, R. T. A Systematic Review of Evidence for a Role of Rest-Activity Rhythms in Dementia. Front Psychiatry 10, 778, doi:10.3389/fpsyt.2019.00778 (2019).

7. Roh, H. W. et al. Associations of rest-activity patterns with amyloid burden, medial temporal lobe atrophy, and cognitive impairment. EBioMedicine 58, 102881, doi:10.1016/j.ebiom.2020.102881 (2020).

8. McGowan, N. M., Goodwin, G. M., Bilderbeck, A. C. \& Saunders, K. E. A. Circadian rest-activity patterns in bipolar disorder and borderline personality disorder. Transl Psychiatry 9, 195, doi:10.1038/s41398019-0526-2 (2019).

9. Leng, Y., Musiek, E. S., Hu, K., Cappuccio, F. P. \& Yaffe, K. Association between circadian rhythms and neurodegenerative diseases. Lancet Neuro/ 18, 307-318, doi:10.1016/S1474-4422(18)30461-7 (2019).

10. Madrid-Navarro, C. J. et al. Multidimensional Circadian Monitoring by Wearable Biosensors in Parkinson's Disease. Front Neuro/ 9, 157, doi:10.3389/fneur.2018.00157 (2018).

11. Musiek, E. S. \& Holtzman, D. M. Mechanisms linking circadian clocks, sleep, and neurodegeneration. Science 354, 1004-1008, doi:10.1126/science.aah4968 (2016).

12. Vallieres, A. \& Morin, C. M. Actigraphy in the assessment of insomnia. Sleep 26, 902-906, doi:10.1093/sleep/26.7.902 (2003). 
13. Sanchez-Ortuno, M. M., Edinger, J. D., Means, M. K. \& Almirall, D. Home is where sleep is: an ecological approach to test the validity of actigraphy for the assessment of insomnia. J Clin Sleep Med 6, 21-29 (2010).

14. McCall, C. \& McCall, W. V. Comparison of actigraphy with polysomnography and sleep logs in depressed insomniacs. J Sleep Res 21, 122-127, doi:10.1111/j.1365-2869.2011.00917.x (2012).

15. Zavada, A., Gordijn, M. C., Beersma, D. G., Daan, S. \& Roenneberg, T. Comparison of the Munich Chronotype Questionnaire with the Horne-Ostberg's Morningness-Eveningness Score. Chronobiol Int 22, 267-278, doi:10.1081/cbi-200053536 (2005).

16. Sadeh, A. The role and validity of actigraphy in sleep medicine: an update. Sleep Med Rev 15, 259267, doi:10.1016/j.smrv.2010.10.001 (2011).

17. Smith, M. T. et al. Use of actigraphy for the evaluation of sleep disorders and circadian rhythm sleepwake disorders: an American Academy of Sleep Medicine clinical practice guideline. Journal of Clinical Sleep Medicine 14, 1231-1237 (2018).

18. Natale, V., Plazzi, G. \& Martoni, M. Actigraphy in the assessment of insomnia: a quantitative approach. Sleep 32, 767-771, doi:10.1093/sleep/32.6.767 (2009).

19. Hauri, P. J. \& Wisbey, J. Wrist actigraphy in insomnia. Sleep 15, 293-301, doi:10.1093/sleep/15.4.293 (1992).

20. Williams, J. M. et al. Validity of Actigraphy in Young Adults With Insomnia. Behav Sleep Med 18, 91106, doi:10.1080/15402002.2018.1545653 (2020).

21. Musiek, E. S. et al. Circadian Rest-Activity Pattern Changes in Aging and Preclinical Alzheimer Disease. JAMA Neuro/ 75, 582-590, doi:10.1001/jamaneurol.2017.4719 (2018).

22. Palagini, L. et al. Insomnia, sleep loss, and circadian sleep disturbances in mood disorders: a pathway toward neurodegeneration and neuroprogression? A theoretical review. CNS Spectr, 1-11, doi:10.1017/S1092852921000018 (2021).

23. Rumble, M. E. et al. An exploratory analysis of the association of circadian rhythm dysregulation and insomnia with suicidal ideation over the course of treatment in individuals with depression, insomnia, and suicidal ideation. J Clin Sleep Med 16, 1311-1319, doi:10.5664/jcsm.8508 (2020).

24. Mascaro, L., Phillips, A. J. K., Clark, J. W., Straus, L. D. \& Drummond, S. P. A. Diurnal Rhythm Robustness in Individuals With PTSD and Insomnia and The Association With Sleep. J Biol Rhythms 36, 185-195, doi:10.1177/0748730420984563 (2021).

25. Bonnet, M. H. Evidence for the pathophysiology of insomnia. Sleep 32, 441-442, doi:10.1093/sleep/32.4.441 (2009).

26. Levenson, J. C., Kay, D. B. \& Buysse, D. J. The pathophysiology of insomnia. Chest 147, 1179-1192, doi:10.1378/chest.14-1617 (2015).

27. Choi, S. J., Suh, S., Ong, J. \& Joo, E. Y. Sleep Misperception in Chronic Insomnia Patients with Obstructive Sleep Apnea Syndrome: Implications for Clinical Assessment. J Clin Sleep Med 12, 15171525, doi:10.5664/jcsm.6280 (2016). 
28. Flynn-Evans, E. E. et al. Circadian Phase and Phase Angle Disorders in Primary Insomnia. Sleep 40, doi:10.1093/sleep/zsx163 (2017).

29. Mundey, K., Benloucif, S., Harsanyi, K., Dubocovich, M. L. \& Zee, P. C. Phase-dependent treatment of delayed sleep phase syndrome with melatonin. Sleep 28, 1271-1278, doi:10.1093/sleep/28.10.1271 (2005).

30. Morgenthaler, T. I. et al. Practice parameters for the clinical evaluation and treatment of circadian rhythm sleep disorders. An American Academy of Sleep Medicine report. Sleep 30, 1445-1459, doi:10.1093/sleep/30.11.1445 (2007).

31. Sack, R. L. et al. Circadian rhythm sleep disorders: part II, advanced sleep phase disorder, delayed sleep phase disorder, free-running disorder, and irregular sleep-wake rhythm. An American Academy of Sleep Medicine review. Sleep 30, 1484-1501, doi:10.1093/sleep/30.11.1484 (2007).

32. Kim, L. J. et al. Is the chronotype associated with obstructive sleep apnea? Sleep Breath 19, 645-651, doi:10.1007/s11325-014-1070-1 (2015).

33. Witting, W., Kwa, I. H., Eikelenboom, P., Mirmiran, M. \& Swaab, D. F. Alterations in the circadian restactivity rhythm in aging and Alzheimer's disease. Biol Psychiatry 27, 563-572 (1990).

34. Blume, C., Santhi, N. \& Schabus, M. 'nparACT' package for R: A free software tool for the nonparametric analysis of actigraphy data. MethodsX 3, 430-435, doi:10.1016/j.mex.2016.05.006 (2016).

35. Refinetti, R., Lissen, G. C. \& Halberg, F. Procedures for numerical analysis of circadian rhythms. Biol Rhythm Res 38, 275-325, doi:10.1080/09291010600903692 (2007).

36. Johns, M. W. A new method for measuring daytime sleepiness: the Epworth sleepiness scale. Sleep 14, 540-545, doi:10.1093/sleep/14.6.540 (1991).

37. Bastien, C. H., Vallieres, A. \& Morin, C. M. Validation of the Insomnia Severity Index as an outcome measure for insomnia research. Sleep Med 2, 297-307, doi:10.1016/s1389-9457(00)00065-4 (2001).

\section{Figures}



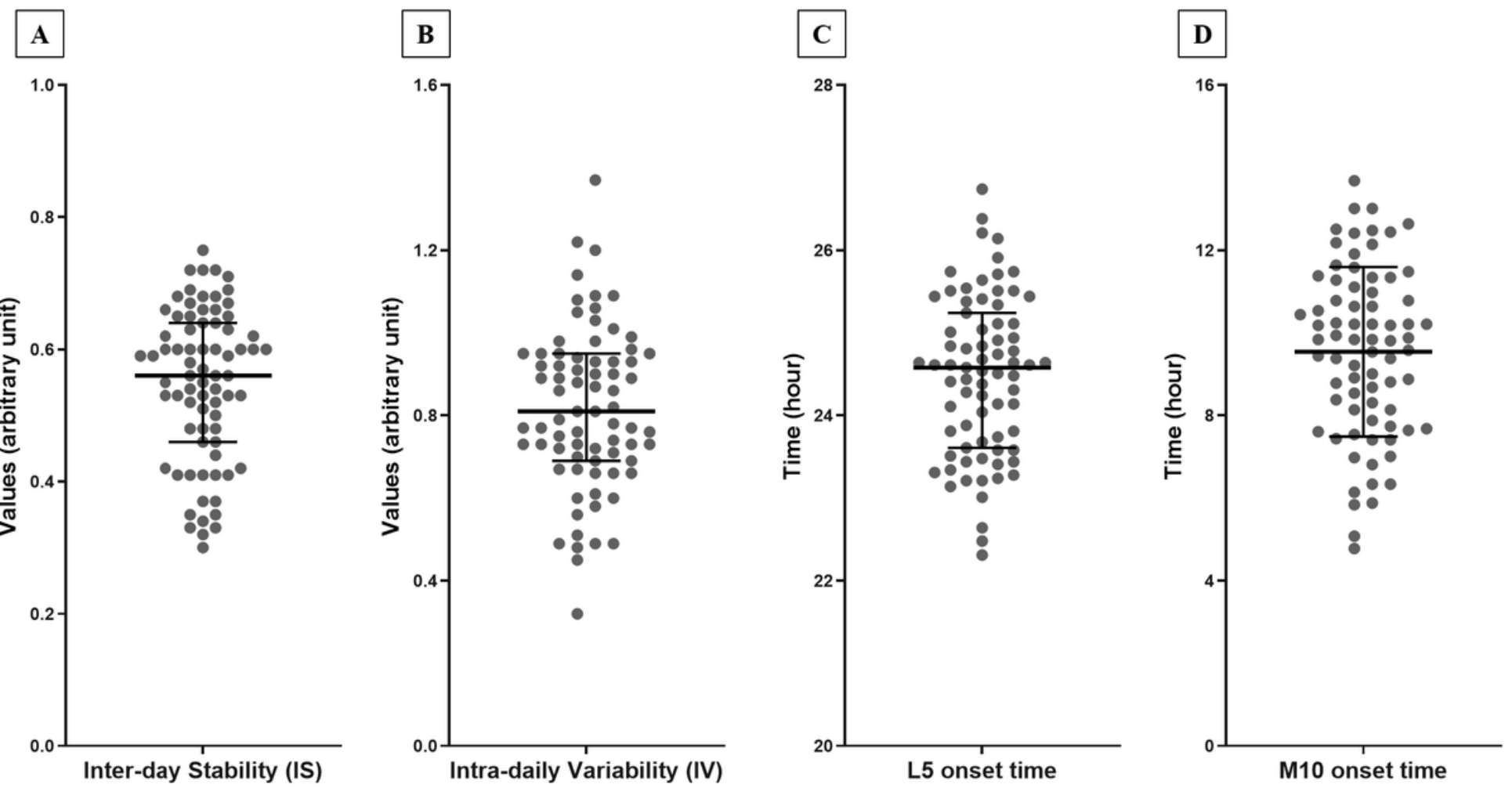

M10 onset time

Figure 1

Distributions of rest-activity patterns and circadian phase variables. Rest-activity pattern variables are expressed as arbitrary units. Circadian phase variables are expressed as time units. Circles indicate patients. Bars in the middle indicate medians, while error bars indicate interquartile range. IS, inter-daily stability; IV, intra-daily variability; L5 onset time, least active 5-h onset time; M10 onset time, most active 10-h onset time. 
A Group by rest-activity pattern

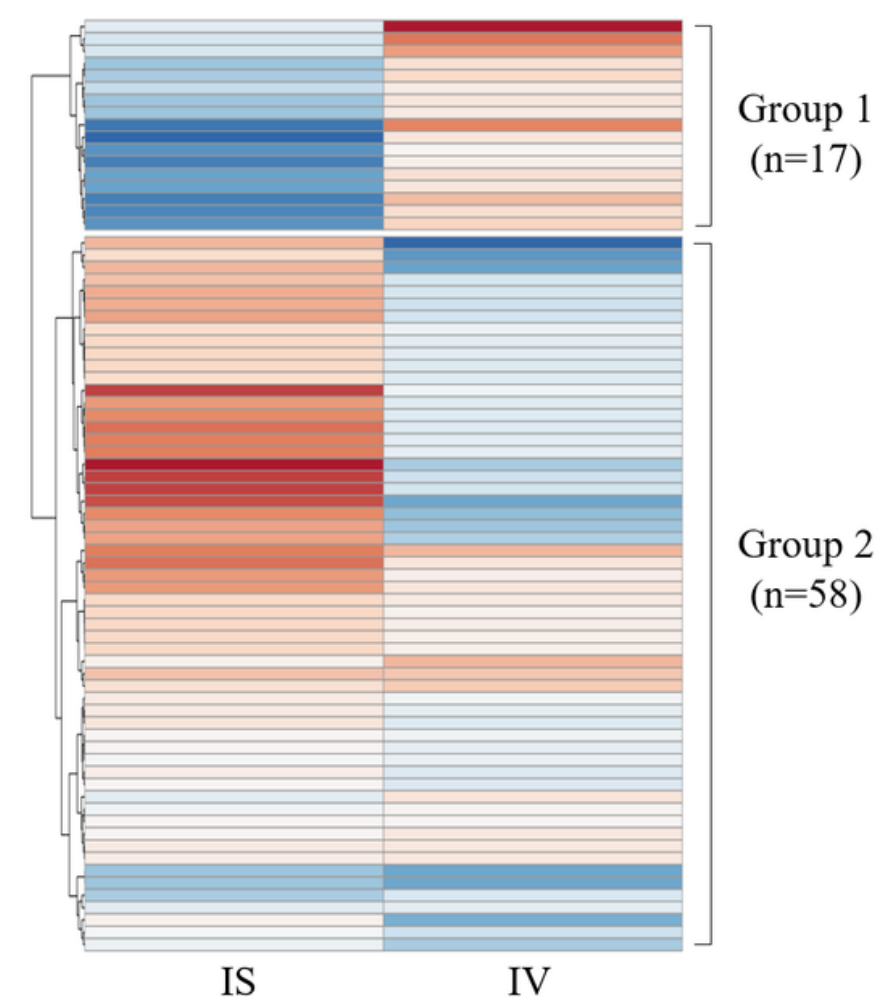

B Group by circadian phase
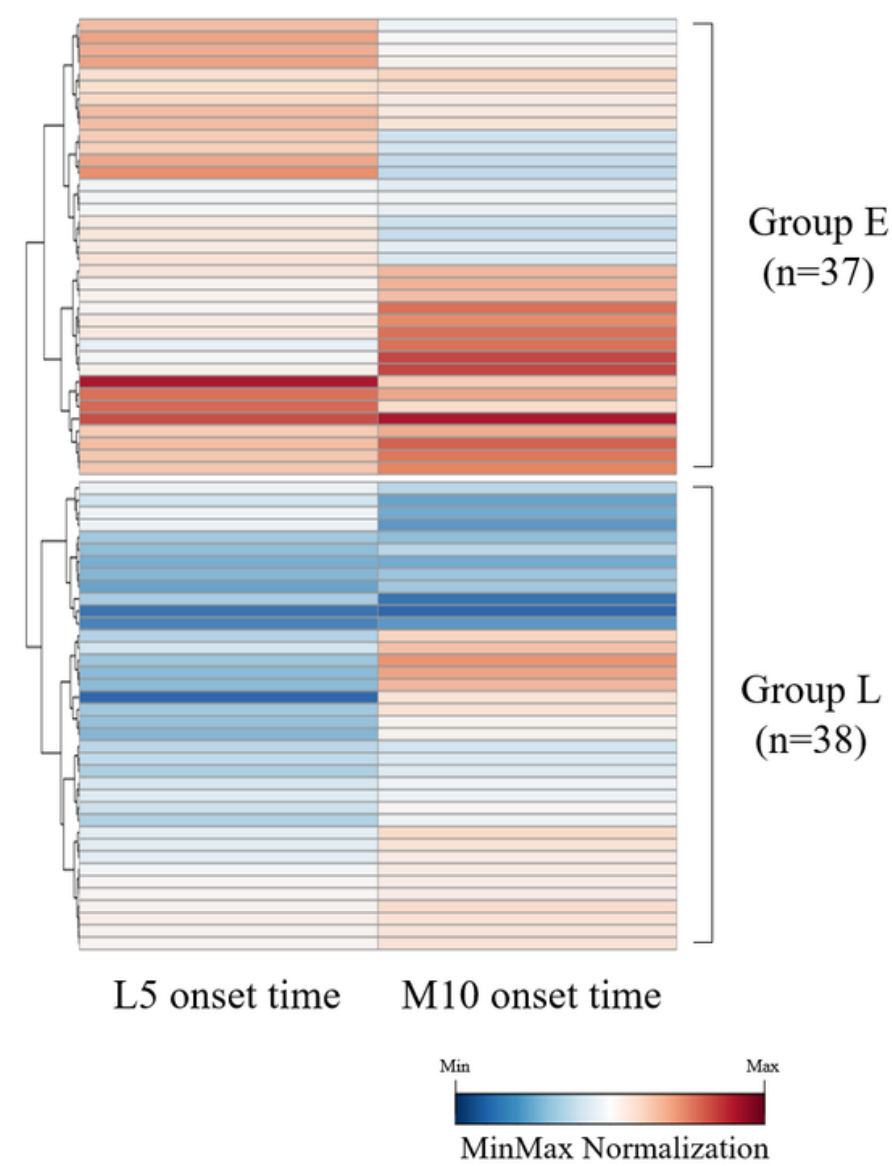

Figure 2

Data-driven group classification of participants based on actigraphy-derived rest-activity patterns and circadian phase. Hierarchical clustering analysis was performed for the data-driven group classification of the participants. IS, inter-daily stability; IV, intra-daily variability; L5 onset time, least active 5-h onset time; M10 onset time, most active 10-h onset time.

A

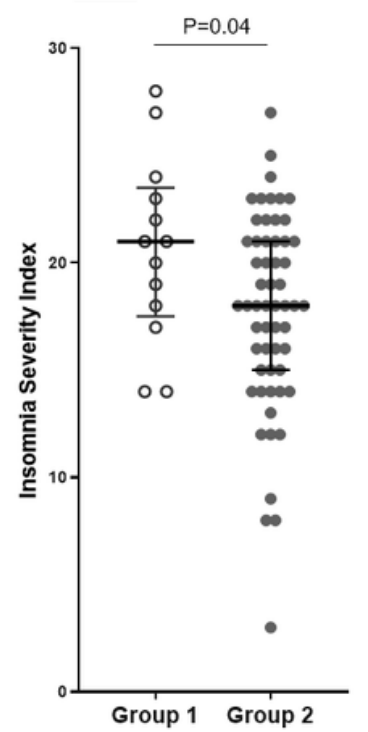

B

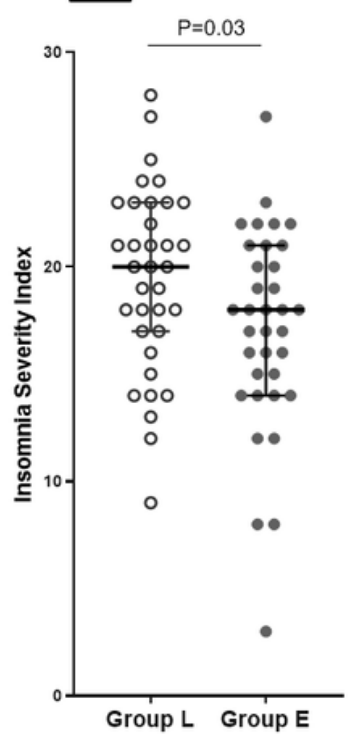

C

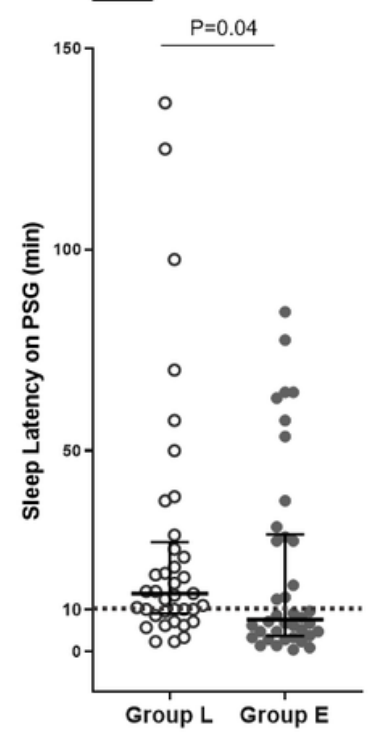

D

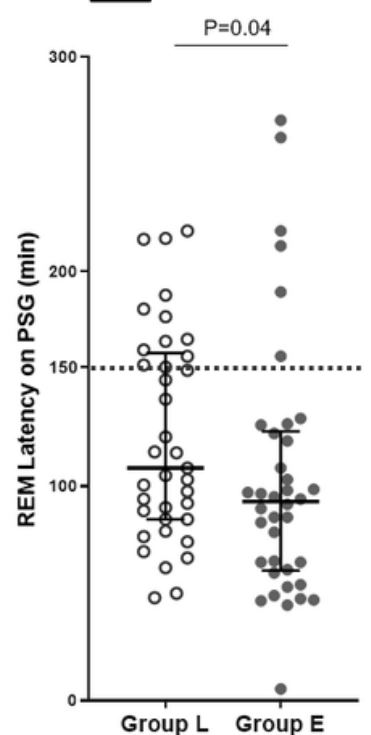

E

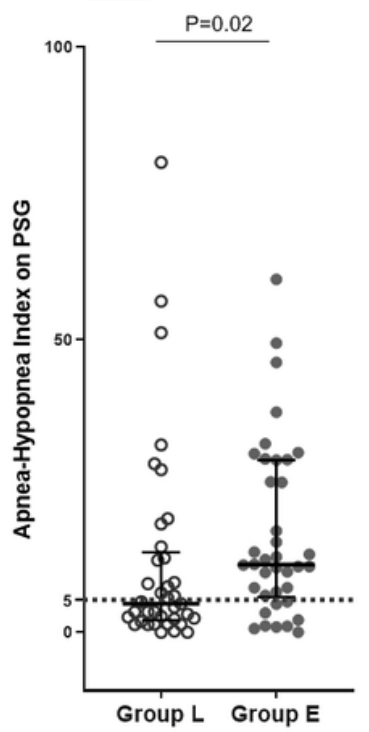




\section{Figure 3}

Differences in clinical symptom scores and PSG parameters between groups according to rest-activity patterns and circadian phase. Group 1 (low IS and high IV), Group 2 (high IS and low IV), Group L (late phase), and Group E (early phase). Student's t-test was performed to analyze normally distributed continuous variables (ISI), and the Mann-Whitney U test was conducted to analyze non-normally distributed continuous variables (sleep latency and $\mathrm{AHI}$ ). Five participants did not complete the insomnia severity index $(n=70)$. Circles indicate patients. Bars in the middle indicate medians, while error bars indicate interquartile range. Dotted lines in Figure $3 c, 3 d$, and $3 e$ indicate the conventional cut-off points for each PSG parameter. AHI, apnea-hypopnea index; IS, inter-daily stability; IV, intra-daily variability; L5 onset time, least active 5-h onset time; M10 onset time, most active 10-h onset time. 